\title{
Multimorbidity Burden in Rheumatoid Arthritis: A Population-based Cohort Study
}

\author{
Tina M. Gunderson ${ }^{1}$ (D), Elena Myasoedova ${ }^{2}$ (D), John M. Davis III $^{3}$, and Cynthia S. Crowson ${ }^{2}$ (i)
}

\begin{abstract}
Objective. To estimate the prevalence and incidence of multimorbidity (MM) in a population-based cohort of patients with rheumatoid arthritis (RA) compared to subjects without RA.

Methods. Between 1999-2013, residents of Olmsted County, Minnesota with incident RA who met the 1987 American College of Rheumatology criteria were compared to age- and sex-matched non-RA subjects from the same population. Twenty-five chronic comorbidities from a combination of the Charlson, Elixhauser, and Rheumatic Disease Comorbidity Indices were included, excluding rheumatic comorbidities. The Aalen-Johansen method was used to estimate the cumulative incidence of MM (MM2+; $\geq 2$ chronic comorbidities) or substantial MM (MM5+; $\geq 5$ ), adjusting for the competing risk of death.

Results. The study included 597 patients with RA and 594 non-RA subjects (70\% female, 90\% White, mean age $55.5 \mathrm{yrs}$ ). At incidence/index date, the prevalence of MM2+ was higher in RA than non-RA subjects (38\% RA vs $32 \%$ non-RA, $P=0.02$ ), whereas prevalence of MM5+ was similar (5\% RA vs. 4\% non-RA, $P=0.68$ ). During follow-up (median 11.6 yrs RA, 11.3 yrs non-RA), more patients with RA developed MM2+ (214 RA vs 188 non-RA; adjusted HR 1.39, 95\% CI 1.14-1.69). By 10 years after RA incidence/ index, the cumulative incidence of MM2+ was $56.5 \%$ among the patients with RA (95\% CI 56.5-62.3\%) compared with $47.9 \%$ among the non-RA (95\% CI 42.8-53.7\%). Patients with RA showed no evidence of increase in incidence of MM5+ (adjusted HR 1.17, 95\% CI 0.93-1.47).

Conclusion. Patients with RA have both a higher prevalence of MM at the time of RA incidence as well as increased incidence thereafter.
\end{abstract}

Key Indexing Terms: comorbidity, rheumatoid arthritis

Rheumatoid arthritis (RA) is characterized by systemic inflammation that can negatively affect multiple body systems, in addition to the joints. Patients with RA are known to have increased risks for cardiovascular disease, interstitial lung disease, osteoporotic fractures, and many other chronic conditions. ${ }^{1,2,3}$ Comorbidities in patients with RA have historically been studied one at a time, but the importance of understanding the co-occurrence of multiple conditions has been recognized in recent years. ${ }^{4}$ The concept of multimorbidity (MM) differs from comorbidity in 2

This work was funded by grants from the National Institutes of Health (NIH), National Institute of Arthritis and Musculoskeletal and Skin Disease (R01 AR46849) and National Institute of Aging (NIA; R01 AG068192). Research reported in this publication was supported by the NIA of the NIH under Award Number R01AG034676. The content is solely the responsibility of the authors and does not necessarily represent the official views of the NIH.

${ }^{I}$ T.M. Gunderson, MS, Department of Health Sciences Research, Mayo Clinic, Rochester; ${ }^{2}$ E. Myasoedova, MD, PhD, C.S. Crowson, PhD, Department of Health Sciences Research, and Division of Rheumatology, Department of Internal Medicine, Mayo Clinic, Rochester; ${ }^{3}$ J.M. Davis III, MD, MS, Division of Rheumatology, Department of Internal Medicine, Mayo Clinic, Rochester, Minnesota, USA.

The authors declare no conflicts of interest.

Address correspondence to Dr. C.S. Crowson, PhD, Mayo Clinic, 2001 st St. SW, Rochester, MN 55905,USA.Email: crowson@mayo.edu.

Accepted for publication February 2, 2021. ways: it is patient-centric, focusing on the patient instead of the index disease of interest, and it is limited to chronic conditions rather than all comorbidities. ${ }^{5}$ This concept is useful in patients with RA because it is reflective of patient complexity and the associated challenges in providing care for patients with RA who have multiple chronic conditions (e.g., the influence of other chronic conditions on RA treatment response). ${ }^{6}$

MM is most commonly defined as the coexistence of 2 or more chronic conditions. However, definitions of 3 or more chronic conditions and definitions based on 5 or more classes of medications have also been used in the literature. ${ }^{7,8}$ Further, there is no agreement on the list of conditions that should be considered when assessing MM. ${ }^{9}$ The most commonly used indices for the general population are the Charlson Comorbidity Index (CCI) and the Elixhauser Comorbidity Index (ECI). ${ }^{10,11}$ The Rheumatic Disease Comorbidity Index (RDCI) and Multimorbidity Index (MMI) were developed for use in patients with rheumatic diseases. ${ }^{4,12}$

$\mathrm{MM}$ is highly prevalent in the general population. ${ }^{13}$ Almost half of the adults in the United States have at least 1 chronic condition, and roughly 1 in 4 have MM, with even higher rates among people aged $>65$ years. ${ }^{14}$ The increasing prevalence of people living with MM stems from multiple causes including obesity, unhealthy diets, sedentary lifestyles, environmental changes, increasing lifespans, an aging population, improved diagnoses and disease detection, drug-disease interactions, 
and disease-disease interactions. ${ }^{15} \mathrm{MM}$ is associated with poor quality of life and high healthcare utilization. ${ }^{16}$

Multiple studies have found a high burden of MM in patients with RA, but the extent of this increase compared to people of similar age and sex without RA has not been well characterized. ${ }^{17,18}$ We aimed to estimate the prevalence and incidence of MM in a population-based cohort of patients with RA compared to subjects without RA.

\section{METHODS}

The study included previously identified Olmsted County, Minnesota residents with incident RA between January 1, 1999, and December 31, 2013. ${ }^{19}$ The complete inpatient and outpatient medical records for each potential case were manually reviewed by an experienced nurse abstractor using the resources of the Rochester Epidemiology Project (REP), and all patients fulfilled the 1987 American College of Rheumatology (ACR) classification criteria for RA. ${ }^{20}$ The REP is a unique medical record linkage system that has provided complete access to inpatient and outpatient medical records of all residents of Olmsted County from all local healthcare providers for more than 50 years. Its history and utility for epidemiological investigations have been described in detail elsewhere. ${ }^{21}$ The incidence date was defined as the earliest date when the patient fulfilled at least 4 of the 1987 ACR criteria for RA.

For each patient with RA, a subject without RA of similar age, sex, and calendar year was randomly selected from the same population to form the non-RA comparison cohort. Each non-RA subject was assigned an index date corresponding to the incidence date of the patient with RA.

Information on patient characteristics was collected at RA incidence/ index date: age, sex, race/ethnicity (White, American Indian/Alaska Native, Asian, Black or African American, Native Hawaiian or other Pacific Islander, > 1 race, or race unknown), smoking status (current, former, or never), BMI, and obesity (BMI $\geq 30 \mathrm{~kg} / \mathrm{m}^{2}$ ). Data on positivity for rheumatoid factor (RF) and anticyclic citrullinated peptide antibody (ACPA) were also abstracted from the medical records. Patients positive for either RF or ACPA were considered seropositive.

Definition of comorbidity, $M M$, and substantial MM. A list of comorbidities relevant to patients in the general population and those with rheumatic diseases was developed by combining lists from the most commonly used indices: CCI, ECI, and RDCI. ${ }^{10,12,22}$ Additional conditions in the MMI were not included because the diagnostic code lists for the MMI were unpublished and could not be obtained from the authors after repeated inquiries. ${ }^{4}$ Diagnostic codes from all healthcare providers in Olmsted County for a period beginning 4 years prior to the RA incidence/index date until last follow-up were used to define 25 chronic medical conditions, including cancer, cerebrovascular disease, chronic pulmonary disease, heart failure, dementia, and myocardial infarction from the CCI; diabetes mellitus, metastatic cancer, valvular disease, liver disease, paralysis, peripheral vascular disorders, renal failure, alcohol and substance abuse, coagulopathy, deficiency anemias, depression, hypertension, hypothyroidism, psychoses, other neurological disorders, pulmonary circulation disorders, and HIV/AIDS from the ECI; and spine, hip, or leg fractures from the RDCI. Diagnostic codes assigned to each comorbidity category were as specified in each index's literature. At least 2 diagnostic codes at least 30 days apart were required to define each comorbidity with the second occurrence of the code used as the diagnosis date of the comorbidity. Rheumatic diseases (i.e., RA) and RA extraarticular manifestations (e.g., interstitial lung disease, secondary Sjögren syndrome, vasculitis) were not included as comorbidities. MM was defined as the presence of $\geq 2$ chronic conditions (MM2+). Due to the high prevalence of hypertension, it was suspected a priori that patients meeting the MM2+ definition would primarily have hypertension plus 1 other condition. A second definition of substantial MM, defined as the presence of $\geq 5$ chronic conditions (MM5+), was examined to capture patients with higher disease complexity and/or disease burden.

Statistical methods. Descriptive statistics (mean, SD, count, percentage) were used to summarize patient characteristics as well as the prevalence of comorbidities, MM2+, and MM5+ at index/incidence date. Comparisons between groups at index/incidence date were performed using chi-square test, Fisher exact test, or Mann-Whitney-Wilcoxon rank-sum test.

Cumulative incidence adjusted for the competing risk of death was estimated for each comorbidity, MM2+, and MM5+ using Aalen-Johansen methods. ${ }^{23}$ These methods are similar to the Kaplan-Meier method with censoring of patients who were still alive at last follow-up. However, patients who died before experiencing MM2+ or MM5+ were appropriately accounted for to avoid overestimation of the rate of occurrence of MM, which can occur if such patients are simply censored at death. These methods also account for any differences in the mortality rates of the RA and non-RA cohorts. Patients who experienced comorbidity, MM2+, or MM5+ prior to RA incidence or index date were excluded from the cumulative incidence and Cox model calculations. Cox proportional hazard models adjusted for age, sex, calendar year of RA incidence/index date, obesity, and smoking were used to compare the rates of development of each comorbidity and MM between groups. A group by calendar year interaction term was used to assess whether trends over time differed by group. A $P$ value of $<0.05$ was considered statistically significant for all analyses. Analyses were performed using R 3.6.2 (R Foundation for Statistical Computing). This study was approved by institutional review boards of Mayo Clinic (IRB \#17-002593) and Olmsted Medical Center (IRB \#017-OMC-17).

\section{RESULTS}

The study included a total of 597 patients with RA and 594 non-RA subjects (Table 1). The RA cohort had a mean (SD) age of 55.5 (15.3) years, was 70\% female and 90\% White, and $40 \%$ were obese at RA incidence. The non-RA cohort had a

Table 1. Characteristics of patients with RA and comparators without RA at incidence/index date.

RA, $\mathrm{n}=597 \quad$ Non-RA, $\mathrm{n}=594$

\begin{tabular}{lcc}
\hline Age, yrs, mean (SD) & $55.5(15.3)$ & $55.4(15.3)$ \\
Sex, female & $419(70)$ & $416(70)$ \\
Race & & \\
$\quad$ Black or African American & $16(3)$ & $17(3)$ \\
White & $538(90)$ & $542(91)$ \\
$\quad$ Other & $43(7)$ & $35(6)$ \\
BMI, kg/m ${ }^{2}$, mean $(\mathrm{SD})$ & $29.2(6.7)$ & $29.3(6.9)$ \\
Obesity, BMI $\geq 30 \mathrm{~kg} / \mathrm{m}^{2}$ & $241(40)$ & $230(39)$ \\
Smoking status & & \\
$\quad$ Never & $313(52)$ & $349(59)$ \\
Former & $191(32)$ & $155(26)$ \\
Current & $93(16)$ & $90(15)$ \\
No. of nonrheumatic comorbidities & & \\
at incidence/index & & \\
0 & $225(38)$ & $245(41)$ \\
1 & $144(24)$ & $161(27)$ \\
2 & $108(18)$ & $91(15)$ \\
3 & $65(11)$ & $49(8)$ \\
4 & $26(4)$ & $23(4)$ \\
$5+$ & $29(5)$ & $25(4)$ \\
\hline
\end{tabular}

Values are expressed as $\mathrm{n}(\%)$ unless otherwise stated. RA: rheumatoid arthritis. 
mean age of 55.4 (15.3) years, was 70\% female and 91\% White, and $39 \%$ were obese at index date. Some differences were noted in smoking status between RA and non-RA groups, primarily among proportions of former smokers (current: 16\% vs 15\%; former: $32 \%$ vs $26 \%$ ). Within the RA cohort, there were $209 \mathrm{RF} /$ ACPA-negative patients and 388 RF/ACPA-positive patients (Table 2). RF/ACPA-positive patients were more diverse $(87 \%$ vs $96 \%$ White, $P=0.002)$. The median length of follow-up was 11.6 (IQR 7.9-15.5) years for RA and 11.3 (IQR 8.1-15.3) for non-RA subjects. During follow-up, 121 patients with RA and 92 non-RA subjects died.

Prevalence and incidence of $M M$. At RA incidence/index date, significantly more patients with RA had MM2+ (228 [38\%] vs 188 [32\%], $P=0.02)$, but not MM5+ (29 [5\%] vs 25 [4\%], $P=0.68$; Table 3). Patients with RA were also significantly more likely to develop MM2+ (10-yr cumulative incidence [95\% CI]: $56.5[51.3-62.3]$ vs 47.9 [42.8-53.7]; HR 1.39 [95\% CI 1.14-1.69]), but showed no significant difference in MM5+ compared to non-RA subjects (26.8 [23.1-31.1] vs 22.1 [18.7-26.2]; adjusted HR 1.17 [0.93-1.47]; Figure 1).

No significant differences were found in incident rates of $\mathrm{MM} 2+$ or MM5+ between RF/ACPA-positive vs -negative patients (Figure 2). The RF/ACPA-negative group showed higher prevalence of $\mathrm{MM} 2+$ and MM5+ at RA incidence (91/209 [44\%] vs $137 / 388$ [35\%], $P=0.05$, and $17 / 209$ [8\%] vs $12 / 388$ [3\%], $P=0.009$, respectively; Table 4 ).

Prevalence and incidence of individual comorbidities. The most common comorbidity in both groups was hypertension (Table 3). When hypertension was excluded from MM2+ and

Table 2. Characteristics of seronegative and seropositive patients with RA at RA incidence date.

\begin{tabular}{lcc}
\hline & $\begin{array}{c}\text { Seronegative, } \\
\mathrm{n}=209\end{array}$ & $\begin{array}{c}\text { Seropositive, } \\
\mathrm{n}=388\end{array}$ \\
\hline $\begin{array}{l}\text { Age, yrs, mean (SD) } \\
\text { Sex, female }\end{array}$ & $56.6(16.0)$ & $54.9(14.9)$ \\
Race & $149(71)$ & $270(70)$ \\
$\quad$ Black or African American & $4(2)$ & $12(3)$ \\
White & $200(96)$ & $338(87)$ \\
Other & $5(2)$ & $38(10)$ \\
BMI, kg/m ${ }^{2}$, mean (SD) & $28.9(6.5)$ & $29.4(6.8)$ \\
Obesity, BMI $\geq 30 \mathrm{~kg} / \mathrm{m}^{2}$ & $74(35)$ & $167(43)$ \\
Smoking status & & \\
Never & $120(57)$ & $193(50)$ \\
Former & $65(31)$ & $126(32)$ \\
Current & $24(12)$ & $69(18)$ \\
No. of nonrheumatic comorbidities & & \\
at RA incidence & & $155(40)$ \\
0 & $70(33)$ & $96(25)$ \\
1 & $48(23)$ & $68(18)$ \\
2 & $40(19)$ & $39(10)$ \\
3 & $26(12)$ & $18(5)$ \\
4 & $8(4)$ & $12(3)$ \\
$5+$ & $17(8)$ & \\
& &
\end{tabular}

Values are expressed as $\mathrm{n}(\%)$ unless otherwise stated. RA: rheumatoid arthritis
MM5+, the increased risk of MM2+ and MM5+ in RA vs non-RA persisted.

Patients with RA showed significantly increased prevalence of hypothyroidism and chronic pulmonary disease at index/ incidence date compared to the non-RA subjects (Table 3). During follow-up, patients with RA showed increased incidence of deficiency anemias, depression, and liver disease compared to non-RA subjects.

$M M$ burden. To estimate the overall comorbidity burden, incidence of MM2+ and MM5+ were also estimated including prevalent cases (Figure 1). For patients with RA, when including prevalent cases, the 10-year cumulative incidence of MM2+ was $73.1 \%$ (95\% CI $69.4-77 \%$; vs $64.4 \%$, 95\% CI $60.3-68.7 \%$ for non-RA subjects) and of MM5+ was 30.3\% (95\% CI 26.6-34.6\%; vs $25.4 \%$, 95\% CI 21.9-29.5\%). Within the RA cohort, when including prevalent cases, the 10-year cumulative incidences for MM2+ and MM5+ in RF/ACPA-positive patients were $71.7 \%$ (95\% CI 67.1-76.6\%) and $26.8 \%$ (22.4-32.1\%) and for RF/ACPA-negative patients were 75.9\% $(69.8-82.5 \%)$ and $36.9 \%$ (30.4-44.8\%).

\section{DISCUSSION}

Patients with RA had a higher prevalence of MM at RA incidence and a higher incidence of MM2+ during follow-up compared to non-RA subjects. RF/ACPA-negative patients with RA had more morbidities at RA incidence than those who were RF/ACPA-positive, but both subgroups had similar incidence of MM2+ and MM5+ after RA incidence.

Comparability across studies is challenging because there is no standardized list of conditions to include when defining $\mathrm{MM}$, though separately both the Charlson and Elixhauser indices are often cited. ${ }^{9}$ Despite this, our findings of a high prevalence of MM in patients with RA are consistent with other reports. A UK-based RA inception cohort reported that the proportion of RA patients with at least 1 comorbidity at RA incidence increased from $29 \%$ in 1990 to $51 \%$ in $2010 .{ }^{24}$ Radner, et al reported $62 \%$ of patients in Brigham and Women's RA Sequential Study (BRASS) had 1 comorbidity and 36\% had at least 2 comorbidities based on a list of 40 morbidities that included the 26 we used and some others with low prevalence (e.g., constipation, hearing loss, psoriasis). ${ }^{4}$ Similar prevalence of $65 \%$ for 1 comorbidity and $34 \%$ for 2 comorbidities were found in the Comorbidities in RA (COMORA) study, which included a list of 17 comorbidities that largely overlapped the list used in this study. ${ }^{17}$ Consistent with our findings, all reports listed hypertension as the most common individual comorbidity. Longitudinal examination of the accumulation of morbidities after RA incidence has not been studied extensively. Consistent with our findings, Yoshida, et al reported an increased accumulation of morbidities in patients with RA compared to those without RA in the Nurses' Health Study, and Nikiphorou, et al reported similar findings in a UK cohort. ${ }^{18,25}$ Using MarketScan data from 2006 to 2015, England, et al showed a greater burden of MM at RA diagnosis and significantly higher accrual over time in patients with RA vs without RA. ${ }^{26}$ 
Table 3. Prevalence at RA incidence/index date and cumulative incidence rate of comorbidities, multimorbidity ( $\geq 2$ comorbidities), and substantial multimorbidity ( $\geq 5$ comorbidities) that developed during follow-up in 597 patients with RA compared to 594 subjects without RA.

\begin{tabular}{|c|c|c|c|c|c|c|}
\hline Comorbidity $^{\mathrm{a}}$ & $\begin{array}{l}\text { No. of Patients Prior to } \\
\text { RA Incidence/Index, } \\
\text { n (\%) }\end{array}$ & $\begin{array}{l}\text { OR }(95 \% \mathrm{CI}) \\
\text { Comparing } \\
\text { Prior Events }\end{array}$ & $\begin{array}{l}\text { No. of Events } \\
\text { After RA I } \\
\text { Incidence/Index } \\
\text { Date, } \mathrm{n}\end{array}$ & $\begin{array}{c}\text { Cumulative } \\
\text { Incidence at } 10 \text { yrs in } \\
\text { x Patients With } \\
\text { RA, } \%(95 \% \mathrm{CI})\end{array}$ & $\begin{array}{c}\text { Cumulative } \\
\text { Incidence at } 10 \text { yrs in } \\
\text { Non-RA Subjects, } \\
\%(95 \% \mathrm{CI})\end{array}$ & $\begin{array}{l}\text { RA vs Non-RA, } \\
\text { HR }(95 \% \text { CI })^{b}\end{array}$ \\
\hline Alcohol abuse & $10(1.7) / 10(1.7)$ & $0.99(0.37-2.69)$ & $17 / 15$ & $3.0(1.9-4.9)$ & $1.9(1.0-3.5)$ & $1.05(0.53-2.11)$ \\
\hline Deficiency anemias & $54(9) / 37(6.2)$ & $1.50(0.95-2.38)$ & $144 / 119$ & $23.9(20.3-28.1)$ & $19.2(16.0-23.2)$ & $1.33(1.05-1.70)$ \\
\hline Cancer & $19(3.2) / 31(5.2)$ & $0.60(0.31-1.11)$ & $65 / 64$ & $9.7(7.5-12.7)$ & $9.9(7.6-13.0)$ & $1.00(0.71-1.42)$ \\
\hline Coagulopathy & $4(0.7) / 0(0)$ & - & $21 / 21$ & $2.9(1.7-4.7)$ & $3.4(2.1-5.4)$ & $1.02(0.56-1.88)$ \\
\hline Dementia & $2(0.3) / 3(0.5)$ & $0.66(0.06-5.8)$ & $25 / 21$ & $3.3(2.0-5.5)$ & $2.4(1.3-4.2)$ & $1.26(0.70-2.26)$ \\
\hline Depression & $61(10.2) / 68(11.4)$ & $0.88(0.6-1.29)$ & $115 / 86$ & $20.8(17.4-24.8)$ & $14.4(11.6-18.0)$ & $1.35(1.02-1.79)$ \\
\hline Diabetes mellitus & $61(10.2) / 63(10.6)$ & $0.96(0.65-1.42)$ & $57 / 56$ & $7.8(5.7-10.6)$ & $10.1(7.7-13.3)$ & $0.96(0.67-1.39)$ \\
\hline Drug abuse & $7(1.2) / 5(0.8)$ & $1.40(0.38-5.62)$ & $17 / 10$ & $2.3(1.3-4.1)$ & $1.5(0.7-3.2)$ & $1.68(0.77-3.68)$ \\
\hline Fracture of spine, hip, or leg & $7(1.2) / 5(0.8)$ & $1.40(0.38-5.62)$ & $26 / 15$ & $4.2(2.8-6.5)$ & $2.0(1.0-3.7)$ & $1.82(0.96-3.44)$ \\
\hline Myocardial infarction & $13(2.2) / 13(2.2)$ & $0.99(0.42-2.35)$ & $27 / 38$ & $4.0(2.6-6.2)$ & $4.8(3.3-7.1)$ & $0.70(0.43-1.15)$ \\
\hline Other neurological disorders & $27(4.5) / 25(4.2)$ & $1.08(0.59-1.96)$ & $111 / 90$ & $15.8(12.8-19.4)$ & $15.6(12.6-19.3)$ & $1.23(0.93-1.63)$ \\
\hline Paralysis & $3(0.5) / 3(0.5)$ & $0.99(0.13-7.46)$ & $10 / 12$ & $1.7(0.9-3.3)$ & $1.7(0.8-3.3)$ & $0.86(0.37-1.99)$ \\
\hline Pulmonary circulation disorder & lers $9(1.5) / 11(1.9)$ & $0.81(0.29-2.17)$ & $38 / 31$ & $5.3(3.7-7.7)$ & $5.2(3.5-7.6)$ & $1.22(0.76-1.97)$ \\
\hline Psychoses & $61(10.2) / 42(7.1)$ & $1.5(0.97-2.31)$ & $69 / 71$ & $11.4(8.8-14.7)$ & $12.9(10.2-16.3)$ & $1.00(0.71-1.39)$ \\
\hline Chronic pulmonary disease & $93(15.6) / 60(10.1)$ & $1.64(1.15-2.36)$ & $104 / 91$ & $19.5(16.1-23.5)$ & $15.6(12.6-19.3)$ & $1.20(0.90-1.59)$ \\
\hline Peripheral vascular disorders & $21(3.5) / 16(2.7)$ & $1.32(0.65-2.73)$ & $117 / 99$ & $16.9(13.9-20.5)$ & $14.9(12.0-18.5)$ & $1.29(0.99-1.69)$ \\
\hline Renal failure & $8(1.3) / 4(0.7)$ & $2.00(0.53-9.14)$ & $54 / 44$ & $7.8(5.8-10.6)$ & $4.9(3.3-7.2)$ & $1.33(0.89-1.99)$ \\
\hline Cerebrovascular disease & $20(3.4) / 18(3)$ & $1.11(0.55-2.25)$ & $38 / 49$ & $5.7(4.0-8.2)$ & $6.9(5.0-9.7)$ & $0.81(0.53-1.24)$ \\
\hline Valvular disease & $25(4.2) / 29(4.9)$ & $0.85(0.47-1.53)$ & $66 / 58$ & $10.8(8.3-13.9)$ & $8.6(6.4-11.5)$ & $1.16(0.82-1.66)$ \\
\hline $\mathrm{MM} 2+$ & $228(38.2) / 188(31.6)$ & $1.33(1.04-1.71)$ & $214 / 188$ & $56.5(51.3-62.3)$ & $47.9(42.8-53.7)$ & $1.39(1.14-1.69)$ \\
\hline MM5+ & $29(4.9) / 25(4.2)$ & $1.16(0.65-2.1)$ & $167 / 139$ & $26.8(23.1-31.1)$ & $22.1(18.7-26.2)$ & $1.17(0.93-1.47)$ \\
\hline
\end{tabular}

${ }^{a}$ HIV/AIDS only occurred in 1 non-RA subject. ${ }^{\text {b }}$ Adjusted for age, sex, calendar year of incidence/index date, smoking, and obesity. MM2+: multimorbidity ( $\geq 2$ comorbidities); MM5+: substantial multimorbidity ( $\geq 5$ comorbidities); RA: rheumatoid arthritis.

Regarding individual chronic conditions, our findings are consistent with others who have previously reported an increased prevalence and/or incidence of hypothyroidism, chronic pulmonary disease, depression, and liver disease in patients with RA. ${ }^{25}$ The lack of increased occurrence of cardiovascular outcomes in this contemporary cohort of patients with RA is consistent with our previous findings and those of others. ${ }^{27,28}$

Reasons for the increased prevalence and incidence of MM in patients with RA are manifold. Inflammation is known to contribute to the development of cardiovascular disease and other comorbidities. ${ }^{29}$ The systemic inflammation that characterizes RA, which is known to begin before RA symptoms manifest, could explain the higher prevalence of $M M$ at RA incidence and could also explain the development of comorbidities after RA incidence. Increased surveillance of patients with RA could also explain some of the increased prevalence of MM in patients with RA, but this might also result from adverse effects of glucocorticoid use or other RA therapies. ${ }^{30}$ Further, the presence of comorbidities complicates RA treatment decisions, as evidence is lacking regarding how to treat real-world patients with comorbidities that may have been excluded from clinical trials of RA therapies or that are listed as contraindications for RA therapies.

The high proportion of seronegative patients in this RA cohort is consistent with previous findings of increasing incidence of seronegative RA. ${ }^{19}$ The higher prevalence of comorbidities at RA incidence in RF/ACPA-negative patients suggests these patients are more medically complex. This complexity may contribute to our earlier findings regarding a delay in diagnosis of RA for RF/ ACPA-negative patients. ${ }^{31}$ Both the high prevalence of comorbidities and the delay in diagnosis of RA may have long-term implications for the treatment and remission of these patients.

These findings underscore the complexity of caring for patients with RA, since a large proportion of these patients have multiple chronic conditions. This complexity makes healthcare decision-making more challenging than in patients without RA. In addition, patients with rheumatic diseases are less likely to receive optimal health maintenance and preventive care services. ${ }^{32}$ Therefore, there is an increasing need for rheumatology care models that provide support for addressing MM and for improved coordination of healthcare between rheumatologists and primary care providers. 

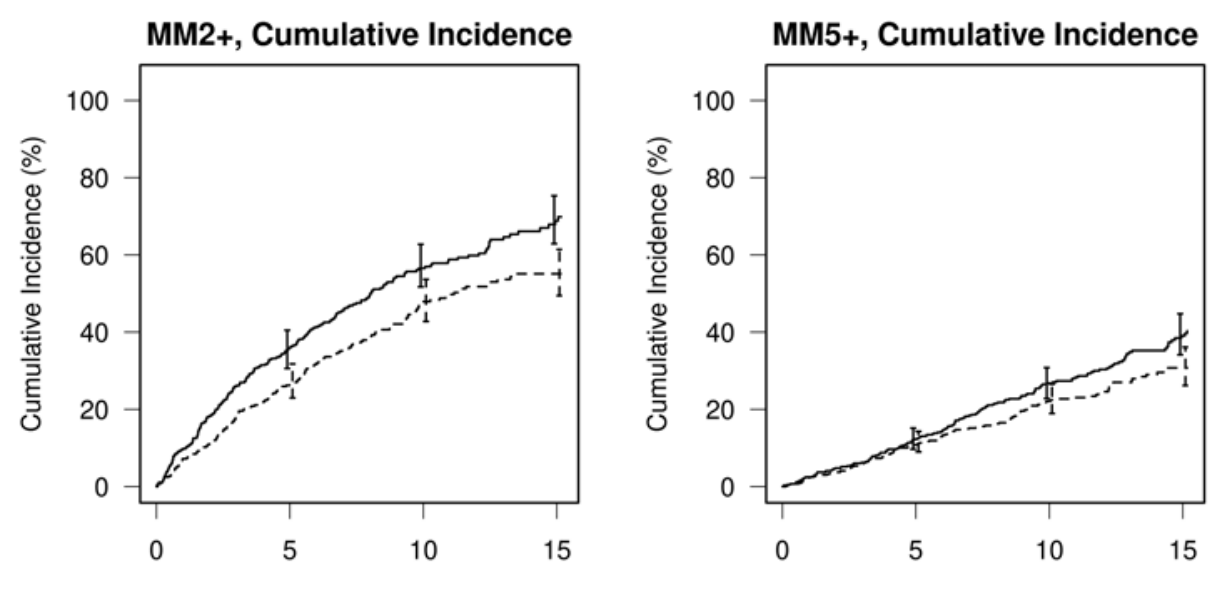

Time since Index/RA Incidence (Yrs) MM2+, Comorbidity Burden
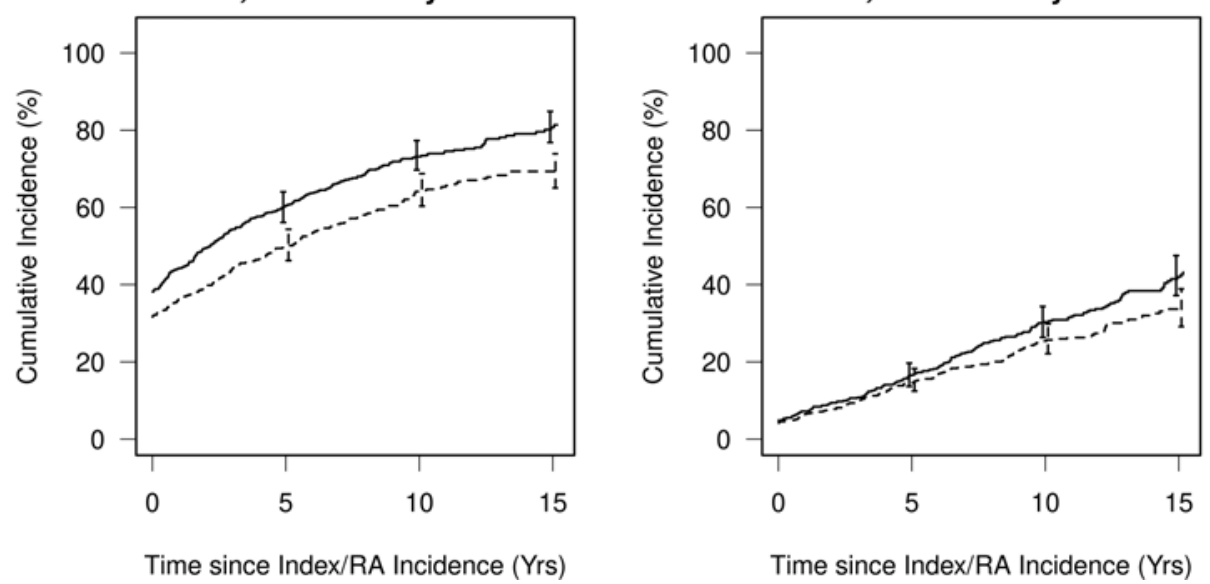

Figure 1. Cumulative incidence (top row) and comorbidity burden (prevalence and cumulative incidence combined; bottom row) of multimorbidity in patients with (solid line) and without (dashed line) rheumatoid arthritis. MM2+ is shown in the left panels and MM5+ in the right panels. MM2+: multimorbidity ( $\geq 2$ comorbidities); MM5+: substantial multimorbidity ( $\geq 5$ comorbidities).

Strengths of this study include the well-characterized, population-based cohort of patients who met classification criteria for RA. The comprehensive resources of the REP facilitated identification of all clinically recognized cases of RA in the population and minimized selection bias. The REP resources also facilitated unbiased selection of non-RA comparators from the same population. Further, the duration of follow-up was long (median $11 \mathrm{yrs}$ ), which allowed assessment of comorbidity accumulation over time. Study limitations include the use of diagnostic codes to define MM and the retrospective study design, which necessitated that the only diagnoses used were those that came to medical attention and were documented in the medical records. The availability of comprehensive medical records from all providers in the community and the focus on chronic comorbidities minimizes the risk of missing comorbidities of interest, but the accuracy of coded diagnoses can be suboptimal. Standard procedures used in administrative claims studies, such as requiring 2 codes at least 30 days apart, were used to improve reliability of the coded diagnoses. Another limitation was the use of a count of chronic conditions to define MM burden. While counts are commonly used in studies of MM, equally weighting conditions does not accurately reflect the differing severity of the conditions. Weighted comorbidity indices have been used to predict various outcomes, but weights also have limitations. For example, weights developed using general population data may not apply to patients with RA, and weighting might partially account for intercondition severity but not intracondition severity. Finally, the limited diversity in the Olmsted County population ( $90 \%$ White) may limit the generalizability of these findings to more diverse populations.

In conclusion, patients with RA already have more comorbid conditions than their non-RA counterparts at RA incidence, and they accumulate more comorbid conditions than non-RA subjects throughout the RA disease course. These findings underscore the challenges faced by the providers who care for these complex patients. More research is needed, both to interpret possible disease clusters or trajectories within the accumulation of these comorbidities and to define strategies to reduce the comorbidity burden for patients with RA. 


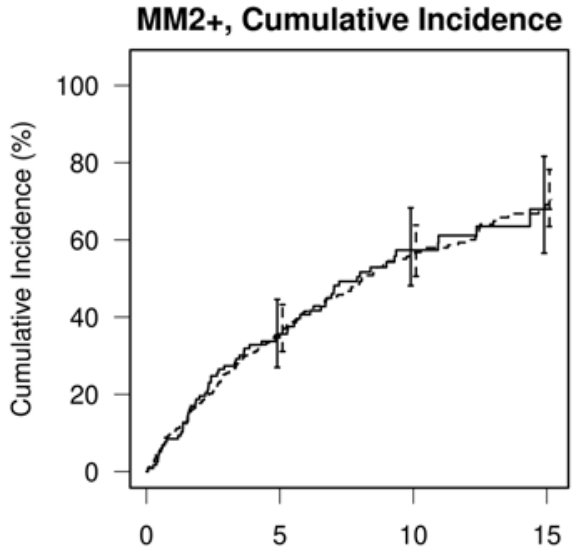

Time since RA Incidence (Yrs) MM2+, Comorbidity Burden

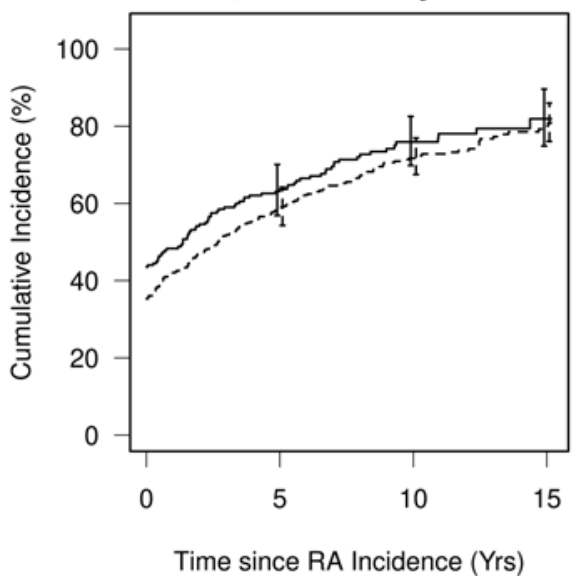

MM5+, Cumulative Incidence

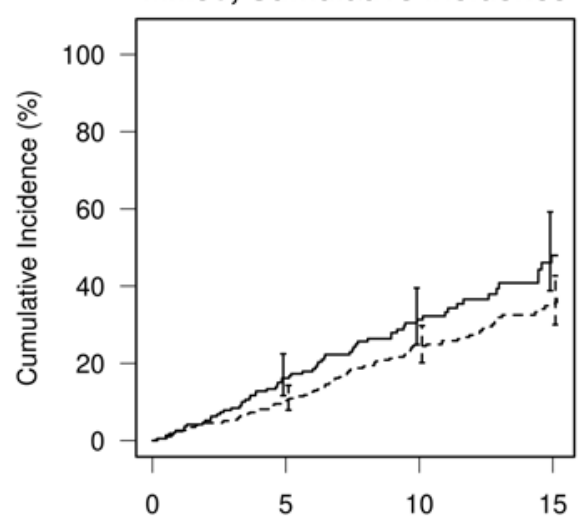

Time since RA Incidence (Yrs) MM5+, Comorbidity Burden

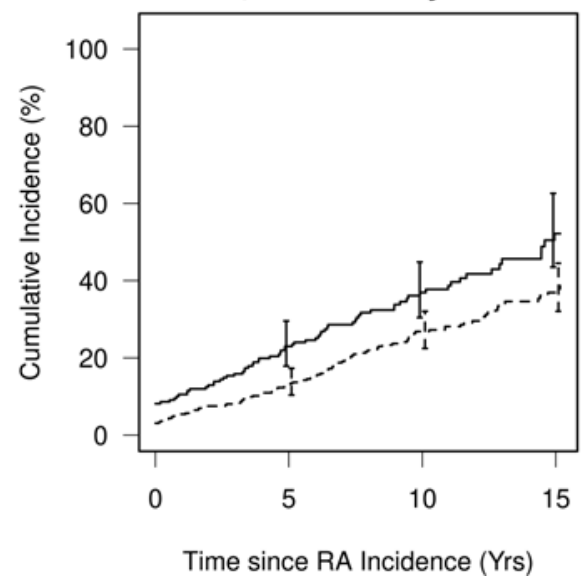

Figure 2. Cumulative incidence (top row) and comorbidity burden (prevalence and cumulative incidence combined; bottom row) of multimorbidity in rheumatoid arthritis patients without (solid line) and with (dashed line) rheumatoid factor and/or anticyclic citrullinated peptide antibody-positivity. MM2+ is shown in the left panels and MM5+ in the right panels. MM2+: multimorbidity ( $\geq 2$ comorbidities); MM5+: substantial multimorbidity ( $\geq 5$ comorbidities).

Table 4. Prevalence at RA incidence/ index date and cumulative incidence rate of multimorbidity ( $\geq 2$ comorbidities), and substantial multimorbidity ( $\geq 5$

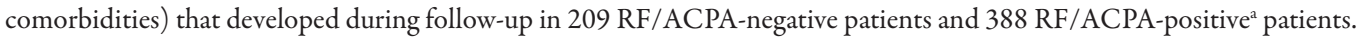

\begin{tabular}{|c|c|c|c|c|c|c|}
\hline Outcome & $\begin{array}{l}\mathrm{N}(\%) \text { of Patients } \\
\text { Prior to RA Incidence } \\
\text { in RF/ACPA, +/- }\end{array}$ & $\begin{array}{c}\text { OR }(95 \% \mathrm{CI}) \\
\text { Comparing Prior } \\
\text { Events }\end{array}$ & $\begin{array}{l}\text { No. of Events After } \\
\text { RA Incidence Date } \\
\text { in RF/ACPA, +/- }\end{array}$ & $\begin{array}{l}\text { Cumulative Incidence } \\
\text { at } 10 \text { yrs in RF/ } \\
\text { ACPA+ Patients, \% } \\
(95 \% \mathrm{CI})\end{array}$ & $\begin{array}{l}\text { Cumulative Incidence } \\
\text { at } 10 \text { yrs in RF/ } \\
\text { ACPA- Patients, \% } \\
\text { (95\% CI) }\end{array}$ & $\begin{array}{c}\text { RF/ACPA+ } \\
\text { vs RF/ACPA-b, } \\
\text { HR (95\% CI) }\end{array}$ \\
\hline MM2+ & $137(35.3) / 91(43.5)$ & $0.71(0.49-1.01)$ & $150 / 64$ & $56.2(50.0-63.2)$ & $57.4(48.1-68.3)$ & $1.00(0.74-1.36)$ \\
\hline MM5+ & $12(3.1) / 17(8.1)$ & $0.36(0.15-0.82)$ & $103 / 64$ & $24.5(20.2-29.7)$ & $31.3(24.8-39.5)$ & $0.83(0.60-1.15)$ \\
\hline
\end{tabular}

${ }^{a}$ RF/ACPA-positive was defined as positive for either RF or ACPA. ${ }^{b}$ Adjusted for age, sex, calendar year of incidence/index date, smoking, and obesity. MM2+: multimorbidity ( $\geq 2$ comorbidities); MM5+: substantial multimorbidity ( $\geq 5$ comorbidities); RA: rheumatoid arthritis; RF: rheumatoid factor/anticyclic citrullinated peptide antibody [positive $(+)$ or negative $(-)]$.

\section{REFERENCES}

1. Maradit-Kremers H, Crowson CS, Nicola PJ, Ballman KV, Roger VL, Jacobsen SJ, et al. Increased unrecognized coronary heart disease and sudden deaths in rheumatoid arthritis: a population-based cohort study. Arthritis Rheum 2005;52:402-11.

2. Bongartz T, Nannini C, Medina-Velasquez YF, Achenbach SJ, Crowson CS, Ryu JH, et al. Incidence and mortality of interstitial lung disease in rheumatoid arthritis: a population-based study. Arthritis Rheum 2010;62:1583-91.

3. Ni Mhuircheartaigh O, Crowson CS, Gabriel SE, Roger VL, Melton LJ 3rd, Amin S. Fragility fractures are associated with an increased risk for cardiovascular events in women and men with rheumatoid arthritis: a population-based study. J Rheumatol 2017;44:558-64.

4. Radner H, Yoshida K, Mjaavatten MD, Aletaha D, Frits M, Lu B, 
et al. Development of a multimorbidity index: impact on quality of life using a rheumatoid arthritis cohort. Semin Arthritis Rheum 2015;45:167-73.

5. Radner H, Yoshida K, Smolen JS, Solomon DH. Multimorbidity and rheumatic conditions-enhancing the concept of comorbidity. Nat Rev Rheumatol 2014;10:252-6.

6. Radner H, Yoshida K, Frits M, Iannaccone C, Shadick NA, Weinblatt M, et al. The impact of multimorbidity status on treatment response in rheumatoid arthritis patients initiating disease-modifying anti-rheumatic drugs. Rheumatology 2015;54:2076-84.

7. Aslam F, Khan NA. Tools for the assessment of comorbidity burden in rheumatoid arthritis. Front Med 2018;5:39.

8. Huntley AL, Johnson R, Purdy S, Valderas JM, Salisbury C. Measures of multimorbidity and morbidity burden for use in primary care and community settings: a systematic review and guide. Ann Fam Med 2012;10:134-41.

9. Johnston MC, Crilly M, Black C, Prescott GJ, Mercer SW. Defining and measuring multimorbidity: a systematic review of systematic reviews. Eur J Public Health 2019;29:182-9.

10. Quan H, Sundararajan V, Halfon P, Fong A, Burnand B, Luthi JC, et al. Coding algorithms for defining comorbidities in ICD-9-CM and ICD-10 administrative data. Med Care 2005;43:1130-9.

11. Elixhauser A, Steiner C, Harris DR, Coffey RM. Comorbidity measures for use with administrative data. Med Care 1998;36:8-27.

12. England BR, Sayles H, Mikuls TR, Johnson DS, Michaud K. Validation of the rheumatic disease comorbidity index. Arthritis Care Res 2015;67:865-72.

13. St Sauver JL, Boyd CM, Grossardt BR, Bobo WV, Finney Rutten LJ, Roger VL, et al. Risk of developing multimorbidity across all ages in an historical cohort study: differences by sex and ethnicity. BMJ Open 2015;5:e006413.

14. Lochner KA, Cox CS. Prevalence of multiple chronic conditions among Medicare beneficiaries, United States, 2010. Prev Chronic Dis 2013;10:E61.

15. Sambamoorthi U, Tan X, Deb A. Multiple chronic conditions and healthcare costs among adults. Expert Rev Pharmacoecon Outcomes Res 2015;15:823-32.

16. Lehnert T, Heider D, Leicht H, Heinrich S, Corrieri S, Luppa M, et al. Review: health care utilization and costs of elderly persons with multiple chronic conditions. Med Care Res Rev 2011;68:387-420.

17. Dougados M, Soubrier M, Antunez A, Balint P, Balsa A, Buch $\mathrm{MH}$, et al. Prevalence of comorbidities in rheumatoid arthritis and evaluation of their monitoring: results of an international, cross-sectional study (COMORA). Ann Rheum Dis 2014;73:62-8.

18. Yoshida K, Lin TC, Wei M, Malspeis S, Chu SH, Camargo CA $\mathrm{Jr}$, et al. Roles of postdiagnosis accumulation of morbidities and lifestyle changes in excess total and cause-specific mortality risk in rheumatoid arthritis. Arthritis Care Res 2021;73:188-98.

19. Myasoedova E, Davis J, Matteson EL, Crowson CS. Is the epidemiology of rheumatoid arthritis changing? Results from a population-based incidence study, 1985-2014. Ann Rheum Dis 2020;79:440-4.
20. Arnett FC, Edworthy SM, Bloch DA, McShane DJ, Fries JF, Cooper NS, et al. The American Rheumatism Association 1987 revised criteria for the classification of rheumatoid arthritis. Arthritis Rheum 1988;31:315-24.

21. St Sauver JL, Grossardt BR, Yawn BP, Melton LJ 3rd, Rocca WA Use of a medical records linkage system to enumerate a dynamic population over time: the Rochester epidemiology project. Am J Epidemiol 2011;173:1059-68.

22. Moore BJ, White S, Washington R, Coenen N, Elixhauser A. Identifying increased risk of readmission and in-hospital mortality using hospital administrative data: the AHRQ Elixhauser Comorbidity Index. Med Care 2017;55:698-705.

23. Gooley TA, Leisenring W, Crowley J, Storer BE. Estimation of failure probabilities in the presence of competing risks: new representations of old estimators. Stat Med 1999;18:695-706.

24. Nikiphorou E, Norton S, Carpenter L, Dixey J, Andrew Walsh D, Kiely P, et al. Secular changes in clinical features at presentation of rheumatoid arthritis: increase in comorbidity but improved inflammatory states. Arthritis Care Res 2017;69:21-7.

25. Nikiphorou E, de Lusignan S, Mallen C, Roberts J, Khavandi K, Bedarida G, et al. Prognostic value of comorbidity indices and lung diseases in early rheumatoid arthritis: a UK population-based study. Rheumatology 2020;59:1296-305.

26. England BR, Roul P, Yang Y, Sayles H, Yu F, Michaud K, et al. Burden and trajectory of multimorbidity in rheumatoid arthritis: a matched cohort study from 2006 to 2015. Ann Rheum Dis 2021;80:286-92.

27. Myasoedova E, Gabriel SE, Matteson EL, Davis JM 3rd, Therneau TM, Crowson CS. Decreased cardiovascular mortality in patients with incident rheumatoid arthritis (RA) in recent years: dawn of a new era in cardiovascular disease in RA? J Rheumatol 2017; 44:732-9.

28. Holmqvist M, Ljung L, Askling J. Acute coronary syndrome in new-onset rheumatoid arthritis: a population-based nationwide cohort study of time trends in risks and excess risks. Ann Rheum Dis 2017;76:1642-7.

29. Innala L, Sjöberg C, Möller B, Ljung L, Smedby T, Södergren A, et al. Co-morbidity in patients with early rheumatoid arthritis inflammation matters. Arthritis Res Ther 2016;18:33.

30. Singh JA, Saag KG, Bridges SL Jr., Akl EA, Bannuru RR, Sullivan $\mathrm{MC}$, et al. 2015 American College of Rheumatology guideline for the treatment of rheumatoid arthritis. Arthritis Rheumatol 2016;68:1-26.

31. Coffey CM, Crowson CS, Myasoedova E, Matteson EL, Davis JM 3rd. Evidence of diagnostic and treatment delay in seronegative rheumatoid arthritis: missing the window of opportunity. Mayo Clin Proc 2019;94:2241-8.

32. Kremers HM, Bidaut-Russell M, Scott CG, Reinalda MS, Zinsmeister AR, Gabriel SE. Preventive medical services among patients with rheumatoid arthritis. J Rheumatol 2003;30:1940-7. 